\title{
A View of Artificial Neural Network Models in Different Application Areas
}

\author{
Kumaravel ArulRaj ${ }^{1}$, *, Muthu Karthikeyan ${ }^{2}$, and Deenadayalan Narmatha ${ }^{3}$ \\ ${ }^{1}$ Department of Mechanical Engineering, Einstein College of Engineering, Tirunelvelli, Tamilnadu, India. \\ ${ }^{2}$ Department of Mechanical Engineering, Raja Rajeshwari College of Engineering, Bengaluru, Karnataka, India. \\ ${ }^{3}$ Department of Electronics and Communication Engineering, Einstein College of Engineering, Tirunelvelli, Tamilnadu, \\ India.
}

\begin{abstract}
Neural network is a web of million numbers of inter-connected neurons which executes parallel processing. An Artificial neural network is a nonlinear mapping structure; an information processing pattern is stimulated by the approach as biological nervous system (brain) process the information. It is used as a powerful tool for modeling the data in the application domains where incomplete understanding of the data relationship to be solved with the readily available trained data. The basic element for this processing pattern is the structure of the data which is the collection of densely interconnected neurons to elucidate the problems. A prominent part of these network is their adaptive nature to "learn by example" just like human substitutes "programming" in resolving the problems. Through learning process, neural net is designed for data classification and prediction where statistical techniques and regression model have been employed. This report is an overview of artificial neural networks in different application areas and it also illustrate the architecture structure formed for the applications. It also provides information about the training algorithm used for certain application.
\end{abstract}

Keywords: Artificial Neural Network and applications, data classification and prediction

\section{Introduction}

Artificial neural network (ANN) as a branch of artificial intelligence have established in a variety of problem-solving areas. The rapid growth of research activity in neural network applications has been accompanied by advancement in the commercial use of ANNs. The concept of artificial neural network is familiarized from the biological neural network which plays a vital role in human body. The human brain comprises of million and million number of neurons that performs a task when existed with the stimulus, it produces an output into other neurons through synaptic connection. Similarly the artificial neurons perform a task by adding the inputs with a transfer function in hidden layer and computing the total value as an output. Based on the structure of the net, an ANN can be single layer or multilayer that is input, feedback or hidden and output as shown in Fig.1. In ANN architecture, training or learning is termed as the programming for adjusting the weights. The learning process can be done by given weights computed from trained data set or by fine- tuning the weights automatically. Trained neural network is expertise in analyzing the information has been furnished with other benefits as fault tolerance, selforganization, real time process and adaptive learning.

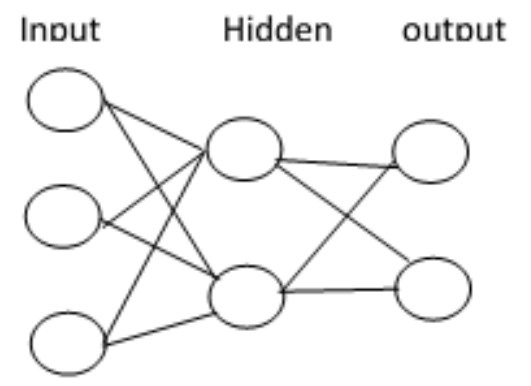

Fig.1 Artificial Neural Network Structure

The main differences between Artificial Neural Networks and Biological Neural Networks (BNN) are described with some parameters as follows;

1. ANN has few kinds of high-speed processing unit whereas BNN has simple and more than 100 kinds of low speed biological neurons is shown in Fig2.

2. The human brain has distributed memory then integrated into processor while ANN having localized memory separated from processor.

3. The computational method in biological 
neurons is occur in neurons, dendrites and synapses with self-learning process in a distributed method while in neural net computing the data in sequential and centralized method.

4. The reliability of the biological network is robust and in $\mathrm{ANN}$ is vulnerable.

5. The operating environment is poorly defined and unconstrained in biological network and for neural net is well defined environment.

6. Error removal capability of human brain is stronger than an ANN by recognizing the error easily.

7. ANN knowledge can be replaceable whereas BNN knowledge is adaptable.

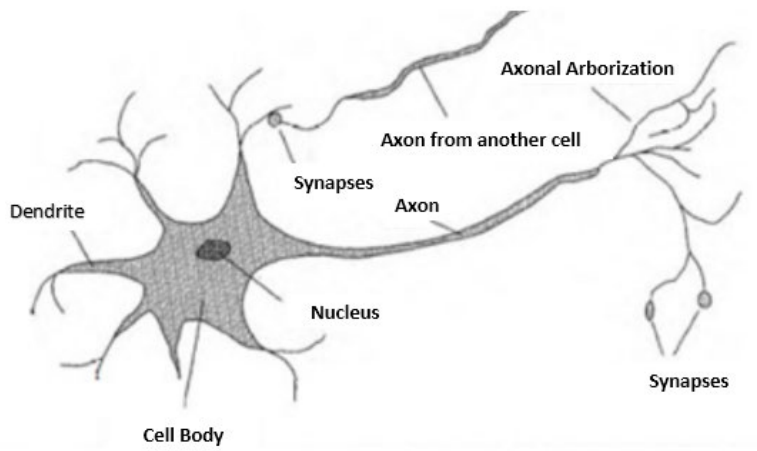

Fig.2 Biological Neuron

To cover wide area of applications, artificial intelligence will enable by integrating the artificial neural network with the expert systems. An ANN has the self-learning capability, the requirement is to provide data and train it; the outcome depends on the structure of the network. The expert system knowledge is restricted with the human knowledge domain; the outcome of this system is subject to how the knowledge has been signified. Both systems have their own characteristics but can cooperate among them and for some issues they overlap in use. [1]

\section{Literature Review}

Rouse viewed that neural network is a web of programs and structure of data which is similar to the human brain function. A neural network comprises of densely interconnected processing unit in parallel each with its own domain of knowledge and data access in its memory. [2]

Kayri observed that in neural network, training algorithm plays an important role in adjusting the weight and minimizing the mean square error between the experimental data and the predicted data output. In the trained data, the error can be reduced to exact threshold level. In this paper, Gradient descent algorithm is used to lessen the mean square error. [3]
Dase et al., presented a review of application of ANN for the prediction of stock market index which is a challenging task. ANN is capable of calculating precise stock index and it also extract the information from the big data thereby it predicts to buy or sell or hold the stock market shares. [4]

Vishnu et al., learned that neural network need not be reprogrammed. When the processing element of the neural net fails, the network can continue its function by parallel processing. ANN is more prevalent in the prediction of outcome from the process parameters by appropriately training the data. [5]

Mitali et al., briefly explained the prediction technique in neural network. This paper reports the different artificial neural network trainings which minimize the mean square error and the steps used in Matlab. ANN is used to increase the prediction accuracy with minimal dependent on experimental data and helps in optimal selection of machining parameter for its optimization. [6]

Rani Pagariya et al., reported the artificial neural network introduction, explained the types of neural network and describe the benefits of neural network with historical background. The mathematical models for the back-propagation algorithm are presented. This paper also investigates the architecture of the neural network. [7]

Foram S. Panchal et al. emphasized that that artificial neural network is more suitable for pattern recognition, signal processing, forecasting, classification and data analysis. To get proper results, neural network is used. It needs trained data, selected architecture and preprocessed data but still the performance is based on the network size. In the neural network, hidden node selection causes overfitting or underfitting problem. This work finds the solution for the selection of hidden node issue by choosing the optimal number of hidden nodes based on similarities of input data after training the network with real data. [8]

Hong Hui Tan et al., explained about the second order optimization technique. This technique offers additional curvature information which reduces the iteration in training the data and attains quick convergence by optimizing the step length in training phase of neural network. It also reviews the techniques involved in second order optimization as follows, Hessian calculation method is appropriate for network training and it is efficient by configuring with conjugate gradient algorithm, Gauss-Newton and Levenberg- Marquardt method uses less memory, with the support of parallel processing computation power per iterations is reduced. The feasibility and optimization technique performance is summarized. [9]

Subhadra et al., proposed a system of 
predicting the heart disease with suitable diagnosis by using multilayer perceptron neural network. The data is trained by the use of back propagation algorithm. The inputs of the network are age, sex, chest, resting blood pressure, serum cholesterol, fasting blood sugar, resting electrocardiograph, heart rate, exercise induced angina, sloping rate and major vessels count. Genetic algorithm, K-mean algorithm are commonly used data mining method for predicting the heart disease. The performance metrics such as sensitivity, specificity, precision and accuracy are calculated for various approaches and proved that the proposed system is proficient in predicting the heart disease. [10]

\section{Aspects and Applications of Neural Networking}

An artificial neural network is an approach which provides the nearer results to human perception and recognition than conventional methods. The distinctive features make neural networks valuable in a variety of applications. One of the characteristics of ANN is that, for an incomplete input, it can bring forth sensible outcomes.

\section{Self-Organizing Traffic Control System}

A four-layer ANN is used to approximate optimal splits of signal phases in traffic control system. The traffic control system is built on two conditions as, constant cycle length over the road network and no offsets between nearby joints. The inputs are control variables such as signal phases split lengths and on inflow links traffic volumes. The output from the neural network are queue length and performance measures. In the training process, by altering the synaptic weights, the targeted outcomes were achieved. [11]

\section{Thrombo Embolic stroke Prediction}

ANN exhibits better performance in the stroke disease prediction by using back propagation algorithm to train the stroke data structure and it has been tested for the types of stroke disease viz. cerebrovascular accident, ischemic stroke, embolic stroke, thrombotic stroke and brain attack. Here neural network helps the doctor in decision making, screening and to double check their diagnosis. Backward stepwise feature selection method is used for input feature selection.

The network structure for the prediction of stroke disease has the twenty input parameters (as shown in Fig. 3 X1 to X20) as Hypertension, Diabetes mellitus, Myocardial infarction, cardiac Failure, Atrial Fibrillation, Smoking, High Blood Cholesterol,
Alcohol abuse, Both right and left Arm and Leg weakness, Speech Slurring, Giddiness, Headache, Vomiting, Memory Deficits, Swallowing Difficulties, Vision loss, isolated vertigo, Hand \& leg Numbness and Dizziness. The network architecture has 20 input nodes, 10 hidden nodes and 10 output nodes.

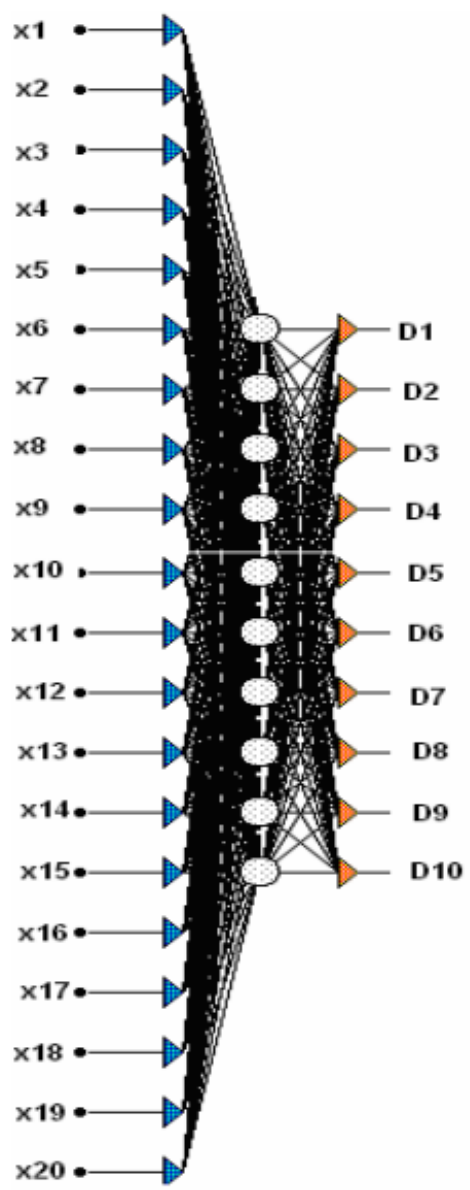

Fig.3 ANN Architecture for Stroke disease Prediction

The types of stroke diseases such as TIA, Left Hemiplegia, Right Hemiplegia, Dysphasia, Monoplegia, Left Hemianopia, Aphasia, Right Hemianthesia, Dysphagia, Quadruplegia are the output parameters (D1 to D10 in Fig3). It helps the doctors to provide earlier diagnosis and better medication to the patient. Neural network provides the improved classification or predication of disease outcome with certain probability. [12]

\section{Shear Wave Velocity Prediction}

Shear wave velocity approximation is the important parameter in the seismic exploration and representation of a carbonate reservoir. To optimize the petro physical parameters multiple regression method is used. The fast training neural network can predict shear wave velocity from carbonate rocks porosity, density and compressional wave velocity. [13] 


\section{Lung Cancer Diagnosing}

In carcinogenesis, neural network has its role in both pre-clinical and post-clinical diagnosis with a high precision rate which supports the clinicians to detect the cancer at its early stage. The back propagation algorithm is used for faster execution and for greater accuracy rate with the trained and test data set obtained from the Lung cancer patients. [14]

\section{Cardiac Arrhythmias Detection}

Cardiac Arrhythmias is the heart disease shows a condition of atypical electrical activity in the heart. It can be detected by using electrocardiogram signal which is a graphical representation of the electrical activity of the heart. The neural network is trained by using Levenberg Marquardt Back propagation algorithm to identify the disease with the trained dataset comprising the QRS complex features as minimum and maximum QRS width, moderate QRS width and the Heart rate [15].

The network architecture for the disease prediction consists of input layer consists of five neurons with tangent sigmoid transfer function and output layer comprises of six neurons which represent the presence of diseases shown in Fig.4 with linear transfer function. Here artificial neural network is used to identify and predict features from highly abnormal ECG and it provides better proficiency for the heartbeats classification according to different arrhythmias.

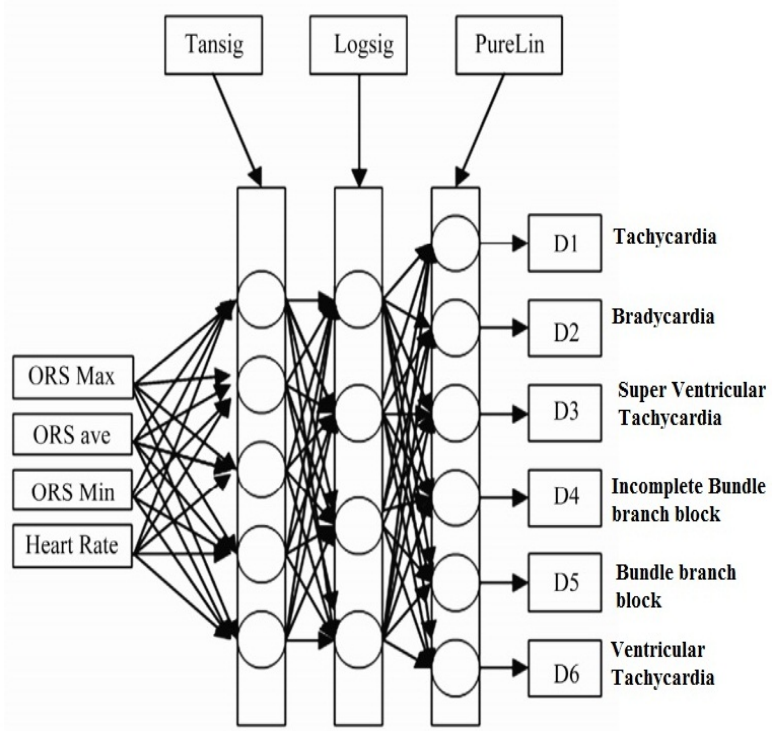

Fig. 4 Network Architecture

\section{Rainfall-run off Prediction}

Rainfall-runoff plays a major role in the water resource management. The inputs for an ANN model are the meteorological variables include rainfall, snowmelt, evaporation and temperature. The artificial neural network is the effective tool to handle the complex problem and has the ability to model rainfall runoff in the arid and semiarid areas where the uneven downpours occur. The impacts of training process and input dimension on rainfall runoff prediction competence of the neural network were applied in many hydrological fields. [16]

\section{Data Mining}

Data Mining is the process of extracting data from the larger database. Data mining can estimate the upcoming trends and supports for decision making. For a data mining task, some of the features like simplicity of the architecture deigning and low computational complexity are achieved by using the artificial neural network. To improve the artificial neural network performance, genetic algorithm is used in neural network design and implements the Neuro- fuzzy system. [17]

\section{Image Processing}

Artificial neural network is used to resolve the real-world problems in the image processing field. In an image processing, the most important step is preprocessing which can be categorized into image construction, restoration and enhancement. Neural network is directly applied to the image features and pixel data. Regression feed forward network is used in preprocessing to reconstruct the images, to suppress noise in image restoration and act as an edge detectors in an image enhancement. In image compression application neural net perform the encoding and in feature extraction applications supervised and unsupervised networks are used to extract the features. The optimization can be done by Hopfield artificial neural network. [18]

\section{Weather Prediction}

Weather forecasting is to determine the state of temperature in future at a given location. For the temperature prediction, three layered back propagation neural network technique is used. It is observed that neural network model produces less error with high efficiency compared with the numerical differentiation method. [19]

Agricultural and industrial field requires accurate weather forecasting which also alert about natural disaster. The input data for the weather prediction in the neural network consists of temperature, air pressure, wind direction, cloudiness, humidity and wind speed collected from meteorological experts. The data are trained using various techniques such as back propagation, optical neural network, radial basis function, regression, and fuzzy ARTMAP [20]

\section{Civil Engineering}


Artificial neural networks have provided an effective tool for various engineering applications including wave-induced seabed instability identification, earthquake- induced liquefaction and tide forecasting. In Tidal level forecasting, to predict the tidal level Ann method is used. Similarly to predict the wave induced and earthquake liquefaction potential ANN produces effective response. [21]

\section{Abrasive Wear Behavior Prediction}

Artificial neural networks approach for the prediction of abrasive wear behavior of carbon fabric reinforced epoxy composite shows that the data predictions are effective when compared with the experimental data. To predict the wear properties, back propagation neural network architecture with different training algorithm is used and compared the results in which Levenberg Marquardt algorithm provides better results.

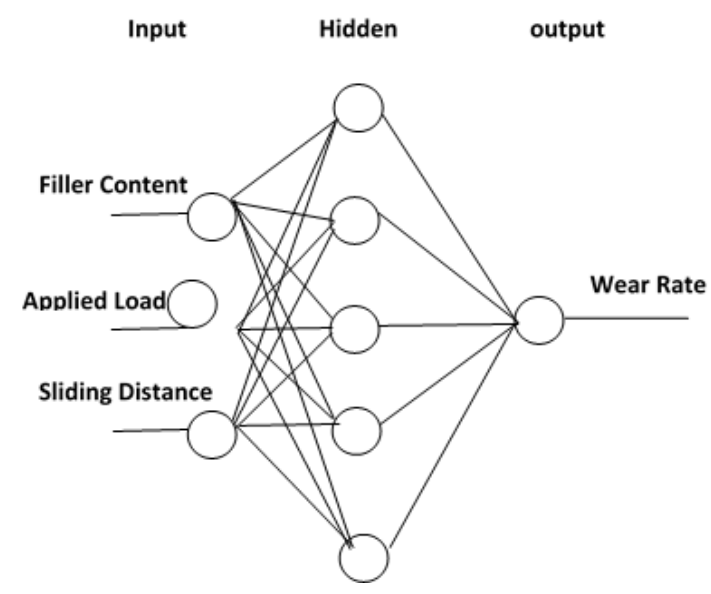

Fig.5 Network Structure for Wear Prediction

In figure.5, Filter content, Applied Load and sliding Distance are the input parameters and wear rate is the output parameter for the Ann architecture. The performance measures such as Sum Squared error, Mean relative error, Mean square error and regression are observed for various algorithms. Trained Ann System helps in estimating the weight loss in the abrasive wear of polymer composites. [22]

\section{Power System}

In multi-area power system, to calculate the transfer capabilities artificial neural networks model was explored. The obtained power transfer capability, voltage magnitudes and voltage angles from repeated power flow method is used for training the input and output patterns of the neural networks. Ann method produces the effective result for the power transfer capability [23]

\section{Medical Diagnosis}

ANN can be used as a classifier and diagnosis tool in medical application because of it intelligent computational techniques. Some of the case studies of ANN in medical applications includes blood flow emboli classification based on trans-cranial ultrasound signals, tissue temperature modeling based on imaging transducer's raw data and identification of ischemic cerebral vascular accident areas based on computer tomography images. In all the cases, ANN shows its high performance and makes it useful for easy diagnosis. [24]

In near future ANN approach in medical field plays a vital role during emergency situations. Due to the environmental change, there will be possibility of more diseases. But nowadays technology has its wide growth, through that self-diagnosis may applicable. Implementing neural networks for medical reasoning provides solutions for common problems by identifying with the symptoms, recognizes the disease and give suggestions for first aid. [25]

ANN has its applications in diagnosis of urinary system disease. Feed forward back propagation act as a classifier to distinguish infected or non-infected urinary system disease such as urinary bladder inflammation and nephritis of renal pelvis. Based on the symptoms, supervised neural networks classify the infected and non-infected person. ANN shows significant results in diagnosis of the disease. [26]

\section{Conclusion}

The fundamental concepts of neural network, the similarities and differences of artificial neural networks with the biological neural network and expert systems were presented. This work reveals the appropriateness and applicability of artificial neural network techniques in various fields. Artificial neural networks are intended to become the powerful tool in enhancing the state of artificial intelligence applications. It also explains the training algorithm and the network structure for various applications of artificial neural network.

\section{References}

1. Rajan Dharwal, Loveneet Kaur, IJST, ISSN 0974-6846, Vol.9, (2016).

2. M. Rouse, Neural Network Definition, available at what's.com Cson, accessed (2015).

3. Kayri Murat, JAMC, Vol. 21, Issue 20, pp. 2- 11, (2016).

4. R.K Dase, D.DPawar . IJMI, ISSN: 0975-2927, 2, 2(2010).

5. Vishnu D. Asal and R. I. Patel, IJSR, Vol.2 (3), (2013).

6. Mitali S Mhatre, Fauzia Siddiqui, MugdhaDongre , Paramjit Thakur, IJSER, ISSN 2229-5518, 6, (2015).

7. Rani Pagariya, MahipBartere, IJAR, 4, 6, (2013).

8. Foram S. Panchal, Mahesh Panchal, IJCSMC, 311, 455-464, (2014).

9. Hong Hui Tan and King Hann Lim, IOP Conference Series: Materials Science and Engineering495, 012003, (2019).

10. K. Subhadra., B.Vikas , (IJITEE) ISSN: 2278- 
3075, 8-5, (2019).

11. T. Nakatsuji and T. Kaku. In Transportation Research Record 1324, TRB, National Research Council, Washington, D.C., (1991).

12. D. Shanthi, G.Sahoo, N.Saravanan,IJBB, 3,1.

13. H. Eskandari., M.R.Rezaee, and. Mohammadnia, CSEG RECORDER, (2004).

14. N.Ganesan, K. Venkatesh, M. A. Rama, A. Malathi Palani, IJCA, 1,6, (2010).

15. Himanshu Gothwal, Silky Kedawat ,JBSE,4, 289-296, (2011).

16. Karim Solaimani, JAES, 5 (6): 856-865, (2009).

17. Nashaat El-KhamisyMohamed, Ahmed Shawky Morsi El-Bhrawy, IOSR-JCE, e-ISSN: 22780661,p-ISSN: 2278-8727, 18, , Ver. III (2016).

18. Rajesh G, A.Muthukumaravel, IJIRCCE,4, 8, (2016)

19. Jyosthna Devi, Syam Prasad Reddy.B, Vagdhan Kumar. K, Musala Reddy.B, Raja.N, IJETT, $3,1,(2012)$.

20. Subhajini A.C, IJWCCCR,4,1,8-18,(2018)

21. Jeng D.S, Cha D.H, Blumenstein.M, ICAIPSIR, (2003).

22. Sudarshan Rao.K, Varadarajan Y.S, Rajendra N (2014), IJEMS, vol 21,pp.16-22.

23. Palukuru Nagendra, sunita Halder nee Dey, Tanaya Dutta (2010), LEJPT, ISSN 1583-1078, Issue 16, p.119-128.

24. Maria Graca Ruano, Antonio E. Ruano (2012), AISC 195, pp 433-451.

25. Sarfaraz Ahmed (2014) IJEDR, ISSN 23219939.

26. Qeerthara Kadhim Al-Shayea, Itedal S.H.Bahia (2010), IJCSNS, vol.10, No.7.

27. ArdeshirFaghri, Jiuyi Hua, Transportation Research Record 1358, Department of Civil Engineering, University of Delaware, Newark.

28. Furqan Yaqui, IJIRSET, 7, 1, (2018).

29. Mishra B.B, Dehuri .S, JCS3 (12); 948-955, (2007). 